\title{
Histologic response after neoadjuvant chemoradiotherapy in locally advanced rectal adenocarcinoma: experience from Sudan.
}

\author{
Ahmed Abd Elrahman Abdalla ${ }^{1}$, Awad Ali M. Alawad ${ }^{2}$, Hussein Abdalla M. Ali ${ }^{3}$
}

1. Department of Surgery, Faculty of Medicine, University of Gezira

2. Department of Surgery, Faculty of Medicine, University of Medical Sciences and Technology, Sudan.

3. Department of Surgery, Faculty of Medicine, Sudan International University, Sudan.

\begin{abstract}
Background: Locally advanced rectal cancer can be down staged by neoadjuvant therapy and the resultant tumor response can be quantified histologically. This study aimed to assess pathological response of neoadjuvant chemoradiation in patients with locally advanced rectal cancers treated in Wad Medani Teaching Hospital (WMTH) and National Cancer Institute (NCI), Wad Medani, Sudan.

Patients and methods: A total of 36 consecutive patients with locally advanced rectal cancer that were managed in WMTH and NCI during the period from 2006-2011 were reviewed. Preoperative pelvic radiotherapy was delivered. Total of 46 Gray were delivered concurrently with 5- fluorouracil (5-FU) on the first and last week of radiation. Total mesorectal excision of the rectal tumour either by anterior or abdominoperineal resections was planned at 6-8 weeks from completion of preoperative treatment. The pathological response to therapy was assessed by histopathology examination of the surgical specimen.

Results: Initial clinical staging of patients revealed $58.3 \%$ of them were stage T3/T4N2M0 and 41.7\% were stage T3N0M0. Down-staging to stage T1/T2N0M0 was found in $36.1 \%$ and stage T3N0M0 in 30.6\%. No response was seen in $8.3 \%$ of cases with stage T3/T4N2M0 while complete clinical response (no residual) was seen in $25.0 \%$. Complete histological response was observed in $13.8 \%$. Positive lymph-nodes metastasis was confirmed in $8.3 \%$ of cases.

Conclusion: Neoadjuvant chemoradiation is a reasonable option for cases of rectal cancer and deserves further evaluation.

Keywords: Neoadjuvant, rectal cancer, chemoradiation, pathological response.

DOI: http://dx.doi.org/10.4314/ahs.v16i3.15

Cite as: Abdalla AAE, Alawad AAM, Ali HAM. Histologic response after neoadjuvant chemoradiotherapy in locally advanced rectal adenocarcinoma: experience from Sudan. Afri Health Sci 2016;16(3): 750-754. DOI: http:// dx.doi.org/10.4314/abs.v16i3.15
\end{abstract}

\section{Introduction}

Rectal cancer accounts for nearly $30 \%$ of all colorectal cancers cases ${ }^{1}$. Surgical resection is the cornerstone of curative therapy. Following a potentially curative resection, the 5-year survival rate varies according to disease extent $^{2,3}$. After establishing the diagnosis and completing the staging work-up, a decision is made whether to pursue immediate resection or administer preoperative chemoradiotherapy(CMRT) ${ }^{4}$.

\section{Corresponding author:}

Awad Ali Mohamed Ahmed,

Department of Surgery, Faculty of Medicine

University of Medical Sciences and Technology, Sudan

Tel: 00249912802545

E-mail: awadali82@hotmail.com
The use of preoperative radiotherapy (RT) combined or not with chemotherapy (CM) has been used in the treatment of rectal cancer for the past two decades and its use gradually increased as adjuvant therapy, especially in T3/ T4 and/or N1/N2 tumors ${ }^{5,6}$. The strategy of performing preoperative instead of postoperative treatment, has the proven advantages of lower acute toxicity ${ }^{7}$, lower total dose of radiation needed ${ }^{8}$ and eventual tumor regression and down-staging to enable curative resection and even sphincter preservation $^{8-13}$.

The objective of this study is to assess the pathological response of neoadjuvant chemo radiation in patients with locally advanced rectal cancers treated in WMTH \& NCI in the period 2006-2011 and to compare our results with the reported standards.

\section{Patients and methods}

This is a retrospective review of patients with locally ad- 
vanced rectal cancer that were managed in WMTH and $\backslash$ or NCI during the period from 2006-2011. In all 36 patients records are revised for symptoms. Digital rectal examination findings for all patients were recorded preoperatively at the combined onco-surgical clinic, and all patients biopsy from the mass were taken as punch biopsies either bed side or during endoscopic examination. Radiological examination was performed evaluating the stage of the tumor and TNM staging system was used.

Neoadjuvant chemo radiation regimens were as follows: Pelvic radiotherapy delivered with total central dose of 46 Gray in 23 sessions. Bolus 5-FU was delivered (400 mg/ $\mathrm{m}^{2}$ ) during the first and last weeks of radiation. 5-fluorouracil was given 30 minutes prior to radiation sessions. All patients had been seen at the combined clinic after 6-8 weeks of radiation. Clinical and radiological assessments were then repeated. Finally, the combined clinic decided on type of surgery according to the initial site of the tumor and response to treatment.

Review of the postoperative histopathology report took place addressing the presence of cancer or viable malig- nant cells, extent of invasion and number of involved lymph nodes, a modified pathologic staging system was used. The Rectal Cancer Regression Grade (RCRG), which simplified the classification to three levels, RCRG 1: the tumor is either sterilized or only microscopic foci of adenocarcinoma remain; RCRG 2: marked fibrosis, but with macroscopic tumor still present; and RCRG 3: little or no fibrosis in the presence of abundant macroscopic tumor. RCRG 1 and 2 were considered to represent significant tumor regression ${ }^{14}$. Report included presence of lymph-nodes and if they were involved or not. Data was introduced and analyzed by computer program (SPSS version 17). To determine the statistical significance of differences, the Pearson test was used and probability test (P. value) with $\mathrm{p}<0.05$ considered as significant.

\section{Results}

The total number of cases was thirty six with female to male ratio of 1.25:1. All patients were seen in combined clinic. More than $55 \%$ of cases have tumor less than $4 \mathrm{~cm}$ from the anal verge (Figure 1).

\section{Figure 1: The distance from the anal verge pre and post Neoadjuvant therapy}

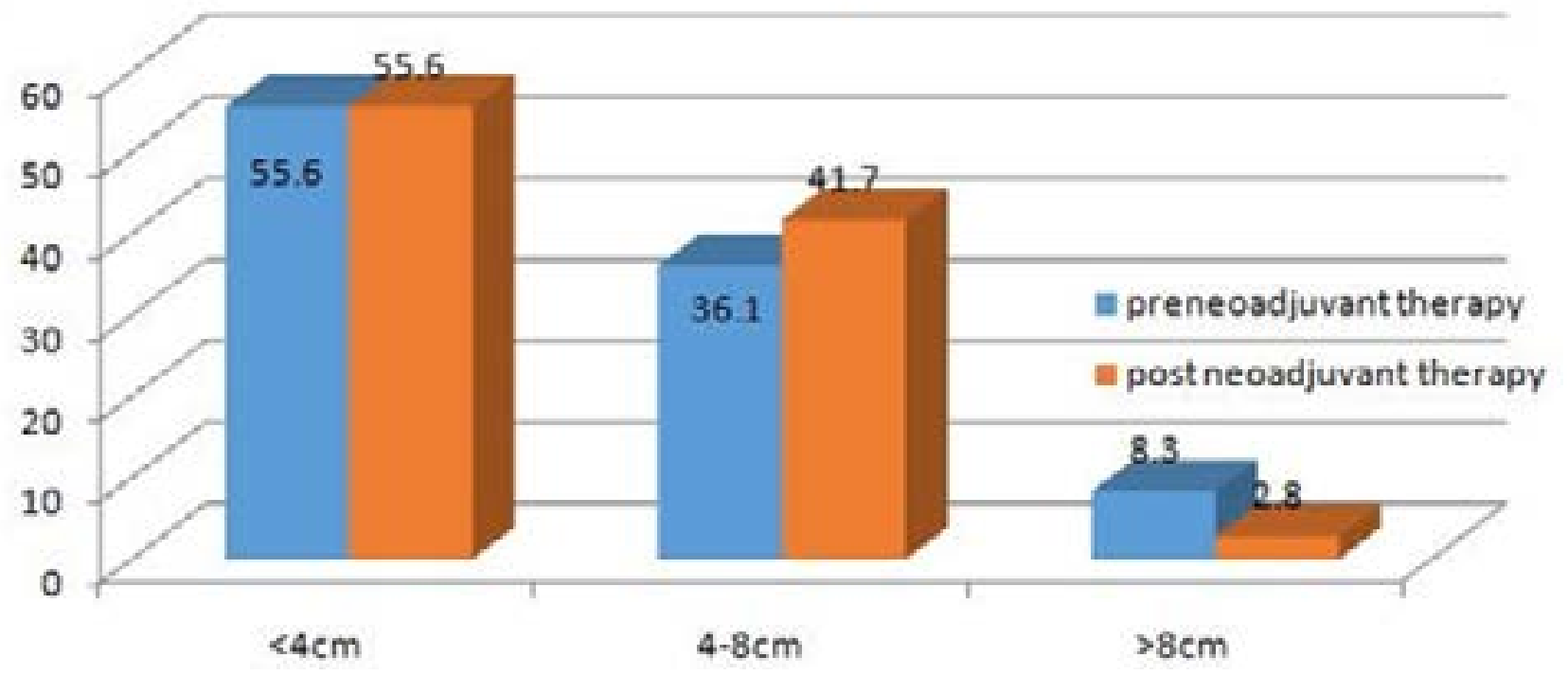

In this study, $97.2 \%(n=35)$ of patients received full course of CMRT and the dose of radiation ranging be- tween 45-50 Gray Pre and post neoadjuvant therapy clinical staging is shown in (Table 1). 
Table 1: Clinical staging using images pre and post CMRT therapy

\begin{tabular}{|c|c|}
\hline $\begin{array}{c}\text { CMRT therapy Clinical } \\
\text { staging using images } \\
\text { (CT/ARI) } \\
\text { Pre-treatment }\end{array}$ & $\begin{array}{c}\text { Clinical staging using } \\
\text { images (CT/MRI) } \\
\text { post-treatment }\end{array}$ \\
\hline 15 stage T3N0M0 & 4 stage TONOM0 \\
& 5 stage T1N0M0 \\
& 4 stage T2N0M0 \\
& 2 stage T3N0M0 \\
\hline 21 stage T3/T4N2M0 & 5 stage TONOM0 \\
& 4 stage T1N0M0 \\
& 9 stage T3N0M0 \\
& 3 stage T4N2M0 \\
\hline
\end{tabular}

In this study $91.7 \%$ of cases underwent APR, $8.3 \%$ cases underwent Anterior Resection (AR) which was done using staplers and one patient offered no surgery. Post neoadjuvant therapy histological assessment showed RCRGI in $41.7 \%$ of cases [of them 5 out of 15 there were com- plete sterilization of the specimen. 13.8\%], RCRGII in $27.8 \%$, and RCRGIII in $30.6 \%$.

Only $8.3 \%$ were found to have metastatic lymph nodes deposits. In cross tabulation between the results of the histology post CMRT and the grade of the tumors, we found that a significant relationship $(\mathrm{P}=0.031)$ between patients grade and response (Table 2 ).

\section{Table 2: Correlation between post Neoadjuvant histological response and tumor grade}

\begin{tabular}{|c|c|c|c|}
\hline $\begin{array}{c}\text { Histopathology } \\
\text { post Neoadjuvant } \\
\text { therapy }\end{array}$ & \multicolumn{3}{|c|}{ Tumor grade } \\
\cline { 2 - 4 } & Grade 1 & Grade 2 & Grade 3 \\
\hline RCRG 1 & 11 & 3 & 1 \\
\hline RCRG 2 & 2 & 6 & 2 \\
\hline RCRG 3 & 2 & 7 & 2 \\
\hline P. value & & 0.031 & \\
\hline
\end{tabular}

\section{Discussion}

Advances in colorectal cancer treatment create a development of a neoadjuvant CMRT which became widely accepted now ${ }^{15}$. Neoadjuvant CMRT is very effective in reducing the tumor mass as seven out of 36 cases showed no palpable mass, per digital rectal examination (DRE), after neoadjuvant CMRT. All cases were amenable for surgery after neoadjuvant CMRT including those who presented with fixed tumor $(69.4 \%)$. This reflected the effectiveness of neoadjuvant CMRT in this study. 
The results of this study can be compared with Dunst et al study done in Germany ${ }^{16}$ who found clinical response rate of $68 \%$ (95\% confidence interval: $57-78 \%$ ), and they have used a total irradiation dose of 50.4-55.8 Gray with conventional fractions. Capecitabine was given at an oral dosage of $825 \mathrm{mg} / \mathrm{m}^{2}$ bid on each day of the radiotherapy period with the first daily dose applied $2 \mathrm{~h}$ before irradiation, followed by surgery 6 weeks later ${ }^{16}$.

In this series $58.3 \%$ of patients $(n=21)$ were found to have stage $\mathrm{T} 3 / \mathrm{T} 4 \mathrm{~N} 2 \mathrm{M} 0$ and post therapy, only 3 cases had this stage $8.3 \%$ and in $41.7 \%$ of patients $(n=15)$ with stage T3N0M0 prior to treatment, the down-staging was seen in (13/15). Radiological complete resolution was observed in $25.0 \%$ of cases. The overall down-staging in this study was observed in (31/36). In comparison with a study done by Rashid A et al they showed down-staging was found in $56.7 \%$ of cases $^{17}$. Duke's university study showed down-staging in $82 \%$ of cases, and this was compatible with our findings ${ }^{18}$.

In a study conducted in Shanghai (2001-2005) published in 2007, 105 patients were studied, of these 13 patients showed complete tumor response after neoadjuvant therapy and they spared the operation ${ }^{19}$. In our study, we were following the case who experienced complete clinical and pathological response, and who remained free since 2009. Pathological complete response which was observed in this study was comparable to the findings of Dunst et $\mathrm{al}^{16}$ they have pathologically complete response was achieved in six patients $(7 \%, 95 \%$ confidence interval: 3-14\%). In another study conducted in Karachi; Rashid A et al found that pathological complete resolution of tumor was achieved in $3.3 \%{ }^{17}$.

In cross tabulation between the results of the histology post CMRT and the grade of the tumors, we found a significant relationship $(\mathrm{P}=0.031)$ between patients grade and response. Eleven out of 15 tumors with grade I showed RCRGI, on the other hand only 3 out of 16 tumors grade 2 showed RCRGII, while only one tumor with grade 3 (out of five) showed complete response RCRGIII. This signifies that the tumor grade may predict the response to treatment (Table 2).

In $8.3 \%$ of our patients there were lymph nodes retrieved in the specimen after surgical resection, this correlates well with the series reported by De laFuente SG et $\mathrm{al}^{20}$, who found fewer total lymph-nodes were retrieved in the neoadjuvant therapy patients compared to those who did not receive preoperatively therapy (Neo 14.6 +/- 0.6 vs. No-Neo 17.2 +/- 1.1)

In conclusion, neoadjuvant chemoradiation in locally advanced rectal cancer in Sudanese patients provide a significant pathological response and it deserves further evaluation.

\section{Acknowledgements}

There was no grant or other financial support for this article.

\section{Conflict of interest}

None to declare.

\section{References}

1. American cancer society. Cancer facts \& figures, Vol 1951. New York: American Cancer Society; 2007

2. Jessup JM, Stewart AK, Menck HR. The national cancer data base report on patterns of care for adenocarcinoma of the rectum, 1985-1995. Cancer 1998; 83:2408.

3. Willett CG, Lewandrowski K, Donnelly S, et al. Are there patients with stage I rectal carcinoma at risk for failure after abdominoperinealresection? Cancer 1992;69:1651-5. 4. Michael J Zinner, Stanley W Ashley. Chapter 25. Cancer of the rectum. In: maingot's abdominal operations, 11th edition. New York: McGraw-Hill;p.2000.

5. Minsky BD. Cancer of the colon, rectum and anus. Annual scientific meeting of the American Therapeutic Radiology and Oncology (ASTRO). Salt Lake City: Educational Session; 202-2003.

6. Kachnic LA, Moughan J, Thomas C, et al. The national practice for patients receivingradiation therapy (RT) for carcinoma of the rectum (RC). Comperative analysis of treatment from 1992-1994 and 1988-1989, Pattern of care studies by academic (AC) vs non-academic (NAC) centres. Int J Radiat Oncol Biol Pbys 2004;57(25):s182.

7. Minsky BD, Cohen AM, Enker WE, Combined modality therapy of rectal cancer: decreased acute toxicity with the preoperative approach. JClin Oncol 1992, 10:1218-24. 8. Frykholm GJ, Isacsson U, Nygard K, et al. Preoperative radiotherapy in rectal carcinoma. Aspects of acute adverse effects and radiation technique. Int J Radiat Oncol BiolPhys 1996, 35:1039-48.

9. Holm T, Singnomklao T, Rutqvist LE, Cedermark B. 
Adjuvant preoperative radiotherapy in patients with rectal carcinoma. Adverse effects during long-term follow-up of two randomized trials. Cancer 1996; 78:968-76.

10. Letschert JGJ, Lebesque JV, Aleman BMP, et al. The volume effect in radiation-related late small bowel complications: results of a clinical study of the EORTC Radiotherapy Cooperative Group in patients treated for rectal carcinoma. Radio ther Oncol 1994; 32:116-23.

11. Swedish rectal cancer trial. Improved survival with preoperative radiotherapy in resectable rectal cancer. $N$ Engl J Med 1997; 336:980-7.

12. Martling A, Holm T, Johansson H. The Stockholm II trial on preoperative radiotherapy inrectal carcinoma: long-term follow-up of a population-based study. Cancer 2001; 92:896-902.

13. Ceelen WP, Van Nieuwenhore Y, Fierens K. Preoperative chemoradiation versusradiation only for stage II and III resectable rectal cancer (review). Cochrane data base for systemic reviews; 2009.

14. Wheeler JM, Warren BF, Mortensen NJ, et al. Quantification of histologic regression of rectal cancer after irradiation: a proposal for a modified staging system. Dis Colon Rectum 2002;45:1051-6.

15. Ahmed A Abdalla, Ahmad A Alshaihk, Daffalla O AbuIdris, MohamedElfatih M. Neoadjuvant chemoradia- tion for rectal cancer: analysis of clinical outcomes for patients treated in Wad Medani TeachingHospitaland National Cancer Institute (Sudan) in the period 2006-2011. Sudan Med J 2012 August;48(2):129-134.

16. Dunst J, Debus J, Rudat V, et al. Neoadjuvant capecitabine combined with standard radiotherapy in patients with locally advanced rectal cancer: mature results of a phase II trial. Strablenther Onkol 2008Sep;184(9):4506. Epub 2008 Sep 19.

17. Rashid A, Ahmed S, Ali M, Fareed M, Bilal M. Role of neo-adjuvant chemoradiation in locally advanced rectal cancer. J Coll Physicians SurgPak 2010 Mar;20(3):17580.

18. Mark W. Onaitis, Robert B. Noone, Matthew Hartwig et al. Neoadjuvantchemoradiation for rectal cancer: analysis of clinical outcomes from a 13-year institutional experience. Ann Surg 2001 June; 233(6):778-85.

19. Yu BM, Zhang M, Wu WQ, et al. Efficacy of neoadjuvantradiochemotherapy in treatment of locally advanced low rectal cancer. ZhonghuaWaiKeZaZhi 2007 Apr; 45(7):445-8.

20. De la Fuente SG, Manson RJ, Ludwig KA, Mantyh CR. Neoadjuvantchemoradiation for rectal cancer reduces lymph-node harvest inproctectomy specimens. J Gastrointest Surg 2008 Oct:45(7):445-8. 